\title{
Type synthesis approach for the 2R1T compliant parallel mechanism with a suitable constrained branch
}

\author{
Yajie Zhou ${ }^{1,2}$, Shihua $\mathbf{L i}^{1,2}$, Jing Sun ${ }^{1,2}$, and $\mathbf{L i} \mathbf{Y i}^{1,2}$ \\ ${ }^{1}$ Parallel Robot and Mechatronic System Laboratory of Hebei Province, \\ Yanshan University, Qinhuangdao 066004, China \\ ${ }^{2}$ School of Mechanical Engineering, Yanshan University, Qinhuangdao 066004, China \\ Correspondence: Shihua Li (shli@ysu.edu.cn)
}

Received: 19 May 2021 - Revised: 1 December 2021 - Accepted: 12 January 2022 - Published: 10 February 2022

\begin{abstract}
In the aerospace field, the precision and stiffness for $2 \mathrm{R} 1 \mathrm{~T}$ ( $\mathrm{R}$ denotes the rotation and the $\mathrm{T}$ denotes the translation) degree of freedom (DOF) space posture adjustment mechanisms are required. Compliant parallel mechanisms (CPMs) with a suitable constrained branch (SCB) have the advantages of high precision and high stiffness. Based on screw theory, a new type synthesis approach for a $2 \mathrm{R} 1 \mathrm{~T}$ compliant parallel mechanism with a suitable constrained branch is proposed. The proposed approach is an improvement of the freedom and constraint topology approach. It combines with other methods, including the rigid-body-replacement method, the principle of symmetry, etc. In order to obtain CPMs with a suitable constrained branch, the criterion for the type synthesis is presented. Using this proposed type synthesis approach, a series of CPMs is obtained. They include, but are not limited to, the existing typical 2R1T CPMs with a suitable constrained branch. Furthermore, it identifies the correctness and effectiveness of the approach by analyzing the DOF of the synthesized mechanism. This approach is also suitable for the type synthesis of 4,5 , and 6 DOF compliant parallel mechanisms with a suitable constrained branch.
\end{abstract}

\section{Introduction}

Compliant mechanisms (CMs) have been used in a variety of applications at micro and macro scales, such as bioengineering (Bhargav et al., 2015), micro electro mechanical systems (MEMSs) (Zhang et al., 2016; Chen and Ma, 2015; Chen et al., 2021), aerospace (S. L. Chen et al., 2018), laser communication (Clark et al., 2016; Cui et al., 2021), and other fields (Yu et al., 2014; Qiu et al., 2018; Y. C. Chen et al., 2018; Qi et al., 2018). They transmit motion/loads by the elastic deformation of materials. This revolutionary change leads to many potential merits, such as reduced part count (up to monolithic configuration), zero backlashes, no need for lubrication, reduced wear, increased reliability, high precision, and compact configuration in comparison with the rigid-body counterparts. However, the applications of CMs are limited because it is hard to make a precision micro-positioning system with both a large workspace and high stiffness at the same time.
Researchers have conducted related studies for parallel mechanisms (PMs) with a suitable constrained branch that have the advantage of high stiffness (Chen et al., 2019; $\mathrm{Li}$ et al., 2020; L. T. Wang et al., 2019). A 4 PUS/UP (P denotes a translational pair, $\mathrm{U}$ denotes a universal pair, and $\mathrm{S}$ denotes a spherical pair) parallel mechanism with a suitable constrained branch is proposed to manufacture complex curved surfaces of large-scale heterogeneous components in the aerospace field (Zhang and Fang, 2018). A 4 SPS/CU (C denotes a cylinder pair) parallel mechanism is proposed. Its kinematics and mechanical are analyzed (Wang et al., 2012). A 5 UPS/PRPU (R denotes a rotational pair) parallel manipulator is proposed. Its performances of kinematics and dynamics are analyzed (Yao et al., 2017). The reference proposes a 3 SPS/RPR parallel mechanism with high stiffness (Hu and Liu, 2020). Compared with their rigid-body counterparts, the compliant parallel mechanisms (CPMs) can provide the merits of both parallel and compliant mechanisms.

The advantages of CPMs compared with their rigid-body counterparts have raised a growing interest in developing the 
methods for their conceptual synthesis. The three main synthesis approaches in this challenging area are the pseudorigid-body model (PRBM) approach (Cannon and Howell, 2005; Moon et al., 2013; Yang et al., 2021), freedom and constraint topology (FACT) approach (Hopkins and Culpepper, 2010a, b; Yu et al., 2011, 2015, 2016), and topology optimization approach (Bowen et al., 2013; Liang et al., 2020; Emmendoerfer et al., 2020). For example, a compliant parallel mechanism with high precision and a wide working area is presented using the new flexure hinges (Kozuka et al., 2012). Based on the FACT approach, a new method for type synthesis of a $2 \mathrm{~T}$ (where $\mathrm{T}$ denotes translation) redundant actuated CPM is proposed (Li et al., 2021). A new 5 DOF (degrees of freedom) grating and splicing CPM is proposed according to the high-precision requirements of large-diameter grating and splicing mechanisms (Shao et al., 2018). Based on the topology optimization approach, a new method considering stress constraints, manufacturing uncertainty, and geometric nonlinearity is proposed (da Silva et al., 2020). A 4 PPR CPM is proposed. Its kinematics and static stiffness are analyzed (Yang et al., 2019).

However, the CPMs with a suitable constrained branch usually are designed by the PRBM approach (Zhang et al., 2011; Yang et al., 2017; Bilancia et al., 2018; Wang and Zhang, 2017). A new 3 SPS/RPR CPM is proposed, and the notch flexible hinges are used (Yun and Li, 2011). Based on the 3 PUS/PU parallel mechanism, a new CPM with a suitable constrained branch is proposed. The 4 DOF hybrid parallel-serial compliant mechanism is presented, and the notch flexure hinges are used (Pinskier et al., 2018). On the one hand, the PRBM approach is based on rigid-parallel mechanisms and is currently unable to solve the problem of topology selection. On the other hand, a plate flexible unit cannot be directly selected to replace a motion pair for the PRBM approach. Compared with the PRBM approach, the new approach has some advantages. The new synthesis approach can obtain new configurations, and the process of the synthesis is easy. As the same time, the plate flexible unit can be directly selected. Compared with the freedom and constraint topology approach, the new approach has also some advantages. The new synthesis approach can obtain the compliant mechanism with high precision and high stiffness by creatively considering the accuracy performance and stiffness performance. Thus, a systematic type synthesis approach for CPM with a suitable constrained branch is proposed. Based on the new synthesis approach, a series of new compliant mechanisms with a large workspace, high precision, and high stiffness are obtained.

The rest of this paper is organized as follows: Sect. 2 provides a survey of the freedom and constraint space and introduces the parasitic displacement and precision. Section 3 addresses the type synthesis conditions and the process for CPMs with a suitable constrained branch. Section 4 obtains the series of the $2 \mathrm{R} 1 \mathrm{~T}$ (where $\mathrm{R}$ denotes the rotation) motion and high-precision and high-stiffness type of CPMs with a

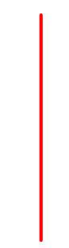

(a)

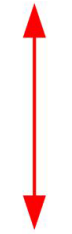

(b)

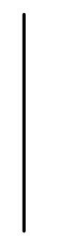

(c)

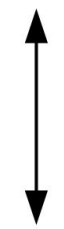

(d)
Figure 1. Freedom and constraint line. (a) Rotation freedom. (b) Translation freedom. (c) Force constraint. (d) Moment constraint.

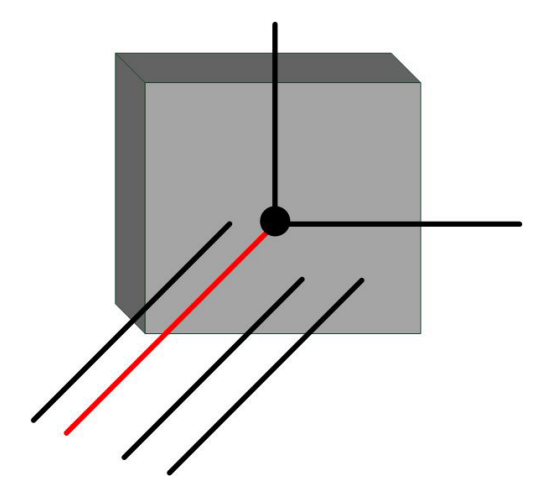

Figure 2. The relationship of the freedom line and all constraint lines.

suitable constrained branch based on the proposed type synthesis approach. Finally, conclusions are drawn.

\section{Theoretic foundation}

\subsection{Freedom and constraint space}

When a rigid body is constrained by several mechanical connections providing $n$ non-redundant constraints, $n$ DOF of the body are removed, correspondingly, while 6- $n$ DOF will remain. In this regard, every constraint wrench in the $n$ system is reciprocal to the twist (6-n) system.

It is clear that the above result complies with Maxwell's principles of constraint. This may be expressed as follows:

$M=6-n$,

where $M$ is the number of DOF.

In order to visualize the relationship between freedoms and constraints in a mechanical system, Blanding (1999) introduced both constraint lines and freedom lines, as shown in Fig. 1. At the same time, he addressed the rule of complementary patterns that states that every freedom line intersects all constraint lines, as shown in Fig. 2 .

Based on the visual constraint-based design method proposed by Blanding, Hopkins and Culpepper extended this method and further proposed a FACT approach (Hopkins and Culpepper, 2011) to achieve a visual type synthesis of com- 
pliant mechanisms. For this purpose, they denoted a collection of commonly used freedom and constraint screw sets as freedom spaces (FSs) and constraint spaces (CSs), respectively, and established luxuriant patterns representing the unique mapping between FSs and their complementary CSs.

\subsection{Parasitic displacement and the precision}

In this paper, parasitic displacement is used to characterize the precision of compliant mechanisms (Zhao et al., 2012). The compliant mechanism with a large parasitic displacement is a low-precision compliant mechanism. The compliant mechanism with a small parasitic displacement is a high-precision compliant mechanism. Compared with the asymmetric compliant mechanism, a symmetrical compliant mechanism has a smaller parasitic displacement. Thus, the symmetrical compliant mechanism has higher precision.

The schematic diagram and a pseudo-rigid-body model of a symmetric CM are shown in Fig. 3. The schematic diagram and a pseudo-rigid-body model of an asymmetric CM are shown in Fig. 4. The reference (Pucheta and Cardona, 2010) can explain the parasitic displacement equation of the flexible beam. Through a superposition operation, the parasitic displacement $\Delta \mathrm{dy}$ of $\mathrm{CM}$ is expressed as follows:

$\Delta \mathrm{dy}=\mathrm{dy} 1+\mathrm{dy} 2$,

where dy $i(i=1,2)$ is the parasitic displacement of a flexible beam.

As shown in Fig. 3b, a symmetric mechanism has two flexible beams, and their parasitic displacements are equal and opposite. Thus, the parasitic displacement relationship for symmetric arrangement of two flexible beams is expressed as follows:

$\mathrm{dy} 1=-\mathrm{dy} 2$.

Similarly, for the asymmetric mechanism, the parasitic displacements of two flexible beams are not equal, and they have a different direction, as shown in Fig. 4b. Thus, the parasitic displacement relationship for a symmetric arrangement of two flexible beams is expressed as follows:

dy $1 \neq-$ dy 2 .

The above results show that the parasitic displacements of the symmetric mechanism can be offset. However, the parasitic displacements of the asymmetric mechanism cannot be offset. As a result, compared with asymmetric CMs, symmetric CMs can achieve higher precision. Thus, the arrangement of the mechanism determines the precision of the mechanism. In order to obtain high-precision CPMs, a symmetric arrangement for the compliant motion branch will be ensured.

\section{Type synthesis approach}

\subsection{Criterion for type synthesis of the CPM with suitable constrained branch}

To ensure that the synthesized mechanism can meet application requirements including motion, precision, and stiffness, a criterion for the type synthesis of the CPM with suitable constrained branch is presented. The following two points should be emphasized for the proposed type synthesis approach.

a. To ensure that the CPM with a suitable constrained branch can achieve the desired motion, the compliant branch need to meet the following conditions:

$M_{1}=M$,

$M_{2} \geq 6$,

where $M_{1}$ is the number of DOF of the compliant suitable constrained branch, and $M_{2}$ is the number of DOF of the compliant active branch. For a $2 \mathrm{R} 1 \mathrm{~T}$ space posture adjustment mechanism as the example, $M$ is equal to three. In order to achieve the desired motion, $M_{1}$ and $M_{2}$ are equal to three and six, respectively. At the same time, in order to reduce errors and simplify the kinematics and dynamics model of the mechanism, the compliant branch needs to be as short as possible.

b. In order to achieve the high precision and high stiffness, the CPM with a suitable constrained branch needs to meet follow conditions:

$n_{\mathrm{s}}>M+1$,

$n_{1}=M$,

where $n_{\mathrm{s}}$ is the number of the compliant branch for a CPM with suitable constrained branch, and $n_{1}$ is the number of identical compliant active branches for a CPM with suitable constrained branch.

First, the number of the compliant active branch and compliant suitable constrained branch is determined, respectively. Then, the appropriate arrangement form is obtained according to the principle of symmetry. The key point for the mechanism is the symmetrical arrangement. The parasitic displacement of the mechanism will be lower, and the high-precision configuration will be obtained by symmetrical arrangement.

For a 2R1T space posture adjustment mechanism as the example, the requirement of high stiffness is obtained. In order to ensure that the mechanism can resist shock and vibration in the rocket during launch, the CPMs with a suitable constrained branch are designed. Compared with the usual CPMs, the CPMs with a suitable constrained branch have higher stiffness. When $M$ is equal to three, in order to achieve 


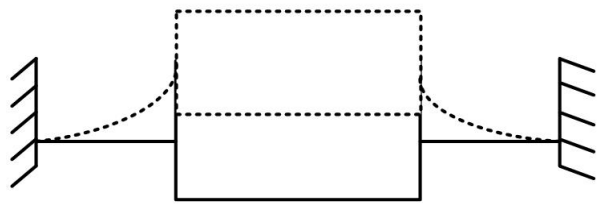

(a)

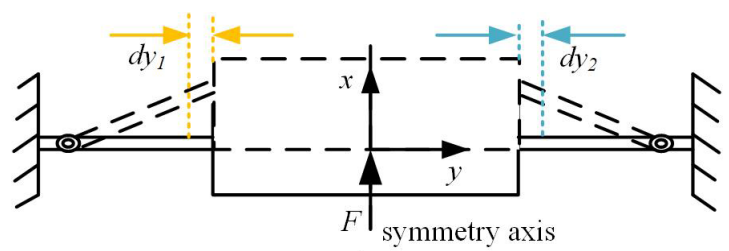

(b)

Figure 3. Symmetrical compliant mechanism. (a) Schematic diagram. (b) PRBM.

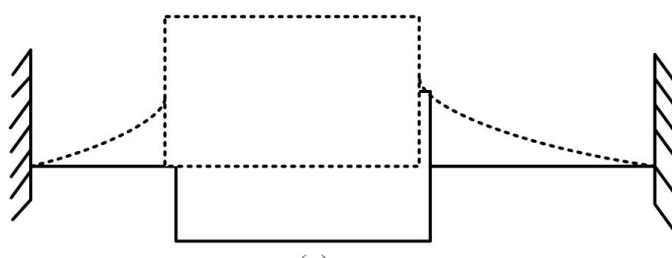

(a)

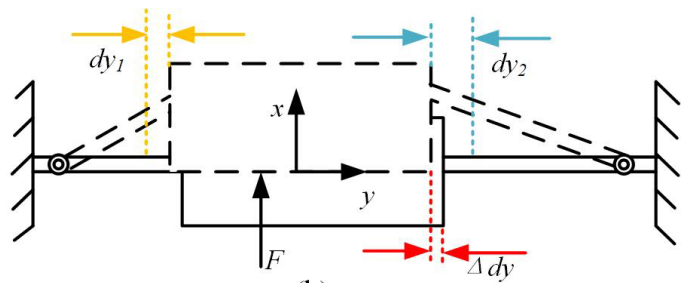

(b)

Figure 4. Asymmetrical compliant mechanism. (a) Schematic diagram. (b) PRBM.

the high precision, the $n_{\mathrm{s}}$ and $n_{1}$ are equal to four and one, respectively. As the same time, in order to reduce the occupied space of the mechanism, the CPMs with a suitable constrained branch need to have as compact a structure and as small a size as possible.

\subsection{Type synthesis process of the CPM with suitable constrained branch}

According to the criterion for the type synthesis of the CPM with a suitable constrained branch, a new synthesis approach is proposed for CPMs with a suitable constrained branch based on the PRBM and FACT methods. According to the given DOF, the number of DOF of the compliant suitable constrained branch, $M_{1}$, is determined, and the number of DOF of the compliant active branch, $M_{2}$, is determined. A compliant branch with a different motion is synthesized based on the PRBM and FACT approaches, respectively. According to the criterion for the type synthesis of the CPM with a suitable constrained branch, the number of compliant active and compliant suitable constrained branches are obtained, respectively. According the principle of symmetry, the new configuration of the symmetrical arrangement mechanism is proposed. The degrees of freedom and motion characteristics of the mechanism are verified by the finite element simulation method. The process for the type synthesis of the CPM with a suitable constrained branch is described in Fig. 5.
Table 1. Some types of the compliant active branches.

\begin{tabular}{|c|c|}
\hline Hinge & Compliant active branch \\
\hline $\mathrm{P}, \mathrm{S}, \mathrm{S}$ & $\underline{\mathrm{PSS}}, \mathrm{SS} \underline{\mathrm{P}}, \mathrm{SP} \underline{\mathrm{P}}$ \\
\hline $\mathrm{P}, \mathrm{U}, \mathrm{S}$ & $\underline{\bar{P}} U S$, SUP,$\overline{S P} \underline{P}$ \\
\hline $\mathrm{R}, \mathrm{S}, \mathrm{S}$ & $\underline{\mathrm{R} S S}, \mathrm{SS} \underline{\mathrm{R}}, \mathrm{SR} \underline{\mathrm{R}}$ \\
\hline $\mathrm{R}, \mathrm{U}, \mathrm{S}$ & $\underline{\mathrm{R} U S,}$ SUR, SRU \\
\hline
\end{tabular}

\section{Synthesis of 2R1T compliant parallel mechanism}

\subsection{Synthesis of compliant active branch}

In order to synthesize the $2 \mathrm{R} 1 \mathrm{~T}$ compliant parallel mechanism, we need to synthesize the compliant active branch based on the proposed synthesis approach. Thus, the motion chain with the 6 DOF need to be synthesized. Besides, according to Sect. 3.1 of the requirements, the short motion chain is selected for the synthesis. Thus, based on the existing 6 DOF serial mechanisms (SMs) in the reference (Lang et al., 2019), these serial mechanisms with only three kinematic pairs are selected as the original branch. This obtains a series of 6 DOF compliant active branches by the rigid-bodyreplacement method, and some of the branches are illustrated in Table 1. For PUS as the example, the P denotes the flexible hinge with connected to the fixed platform, the $\mathrm{S}$ denotes the flexible hinge with connected to the moving platform, and the $\underline{P}$ denotes the flexible hinge as the actuated hinge. 


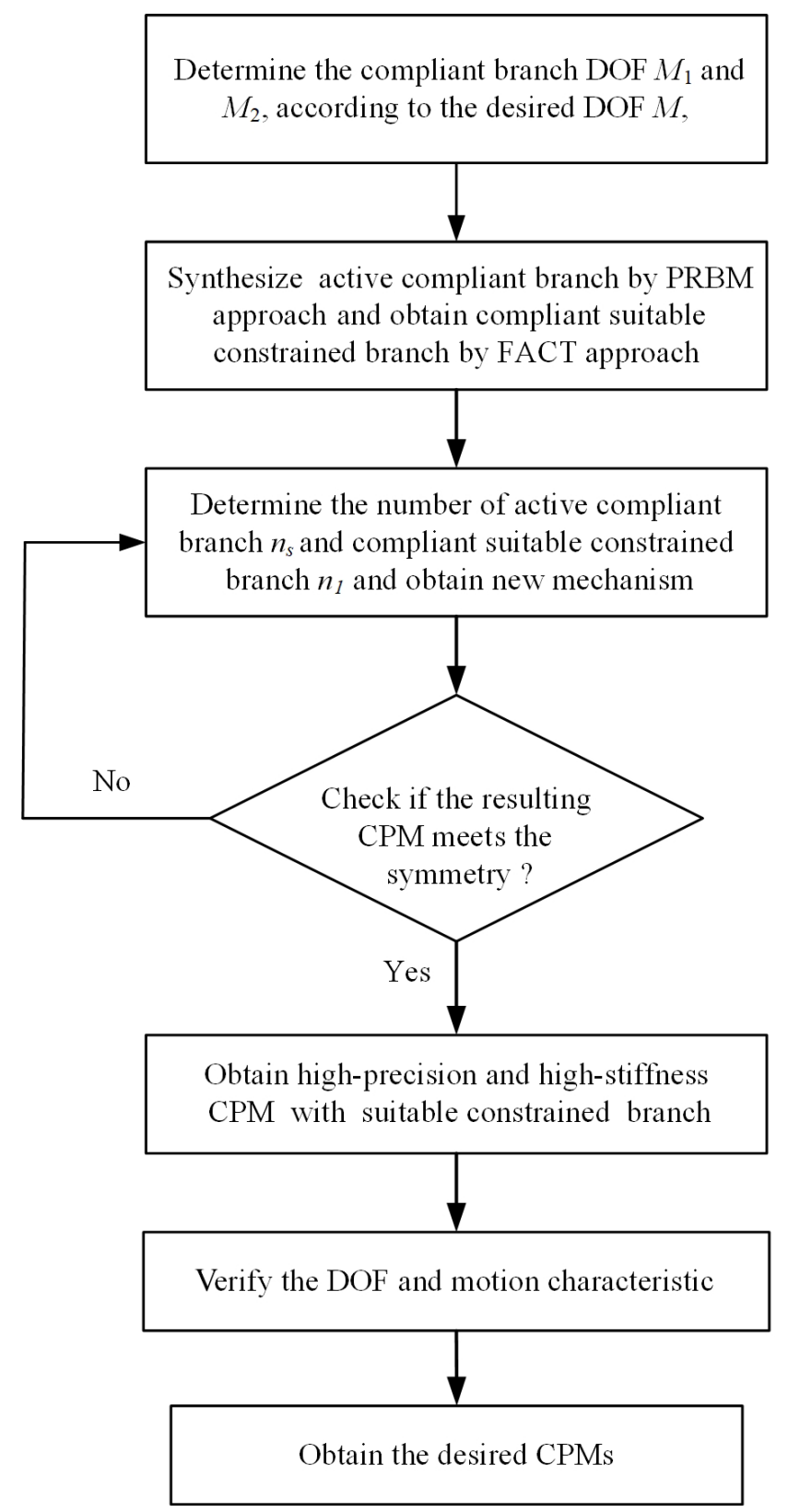

Figure 5. Flow chart for the new approach for type synthesis of the CPM.

\subsection{Synthesis of the compliant suitable constrained branch}

According to the high-stiffness requirement in Sect. 3.1, the CPMs with compliant suitable constrained branches are synthesized to improve the stiffness of the mechanisms. In order to synthesize the $2 \mathrm{R} 1 \mathrm{~T}$ compliant suitable constrained branch, the DOF of the compliant suitable constrained branch need to be determined based on the proposed type synthesis approach. Thus, a 3 DOF compliant suitable constrained branch needs to be synthesized. The total steps

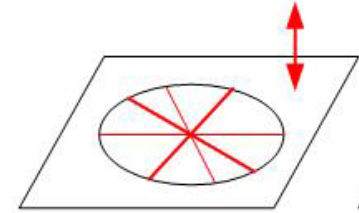

(a)

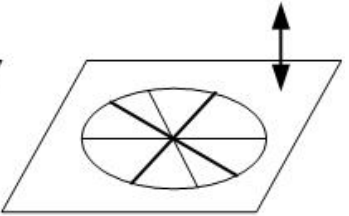

(b)
Figure 6. Freedom and constraint spaces of the 2R1T motion branch. (a) Freedom space. (b) Constraint space.

are performed for the synthesis process of the $2 \mathrm{R} 1 \mathrm{~T}$ compliant suitable constrained branch.

Step 1. Specify the desired freedom space (FS) according to the specifications of a synthesized $\mathrm{CM}$ and specify the reciprocal constraint space (CS) by the dual rule. For the 2R1T mechanisms, the freedom space and constraint space are shown in Fig. 6.

Step 2. Determine all possible reciprocal subspaces representing the constraints from step 1 and generate a complete fundamental building block (FBB) library by decomposing all reciprocal constraint subspaces. For the constraints of the 2R1T motion compliant branch, the FBBs and the simple set symbol are illustrated in Fig. 7.

Step 3. Obtain all possible subspaces equivalent to the FS based on the set operation for FBBs. Note that, in this paper, the union and intersection of the sets are designated by $\cup$ and $\cap$, respectively. For the $2 \mathrm{R} 1 \mathrm{~T}$ motion compliant branch, the set operation is expressed as follows:

$$
\begin{aligned}
L(N, u) & =U\left(N_{1}, n\right) \cup R\left(N_{2}, u\right) \\
& =F\left(N_{1}, u_{1}, n\right) \cup R\left(N_{2}, u_{2}\right) \\
& =R\left(N_{1}, u_{1}\right) \cup R\left(N_{2}, u_{1}\right) \cup R\left(N_{2}, u_{2}\right) \\
& =R\left(N_{1}, u_{1}\right) \cup R\left(N_{2}, u_{2}\right) \cup P(n) \\
& =R\left(N_{1}, u_{1}\right) \cup P(n) \cup R\left(N_{2}, u_{2}\right) \\
& =U\left(N_{1}, n\right) \cup P(n) .
\end{aligned}
$$

Step 4. Establish the mapping relationship between the FBB and the flexure unit, and select the appropriate flexure unit instead of the FBB. For example, $R(N, u)$ represents a rotational degree of freedom (Yu et al., 2010; Lobontiu, 2002). So, a right circular notched flexible unit $\left(\theta_{x}\right)$ can be represented by $R(N, u) . P(n)$ represents a translational degree of freedom (Yang et al., 2021). So, a translational flexible unit $(\delta z)$ can be represented by $P(n) . L(N, n)$ represents a plane constraint with $3 \mathrm{DOF}$. The flexible straight beam unit $\left(\theta_{x}, \theta_{y}\right.$, and $\left.\delta z\right)$ can be represented by $L(N, n)$ (Jia et al., 2015). Some flexible units are shown in Table 2.

Step 5. Obtain the compliant suitable constrained branch via the serial layout. Yu et al. (2010) proposed that the DOF of the flexible straight beam is changed when the dimension parameters of the flexible straight beam are changed (Jia et al., 2015). In this paper, the dimension parameters of the flexible straight beam are selected. The narrow flexible straight 


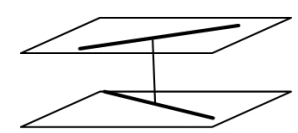

$N(n)$

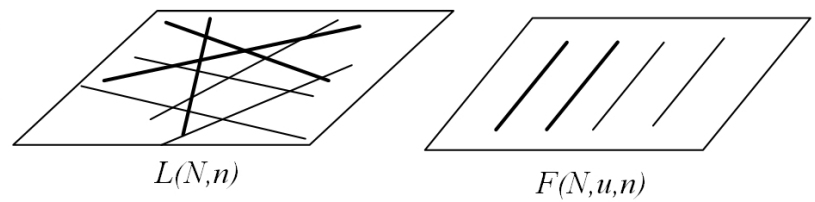

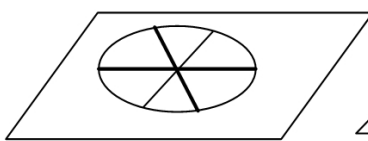

$U(N, n)$

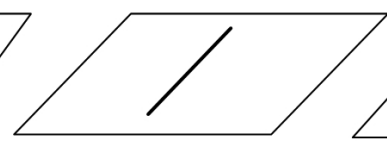

$R(N, u)$
$F(N, u, n)$

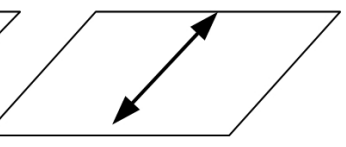

$P(n)$

Figure 7. Fundamental building blocks.

Table 2. Equivalent freedom FBBs of two flexible units.

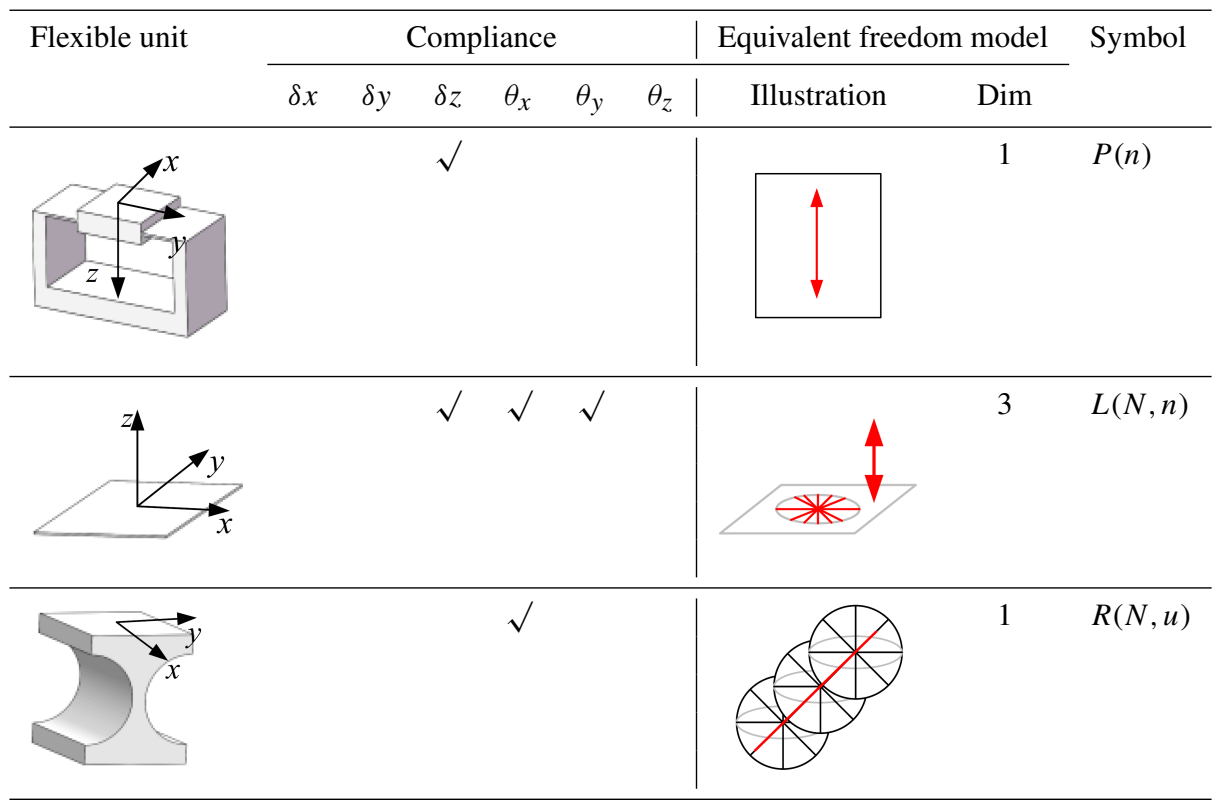

Table 3. Some types of the suitable constraint compliant branches.

\begin{tabular}{ll}
\hline Hinge & Compliant branch \\
\hline L & L1, L2 \\
P, R & PRR, RPR \\
R, U & RU \\
P, U & PU \\
R & RRR \\
\hline
\end{tabular}

beam unit has 3 DOF and can achieve two rotational motions and one translational motion, respectively. Thus, the beam can realize a $2 \mathrm{R} 1 \mathrm{~T}$ motion. For the flexible straight beam, part of the compliant suitable constrained branches are shown in Fig. 8a-b. For the circular notched flexure unit, part of the compliant suitable constrained branches are shown in Fig. 8c.
In Fig. 8, three kinds of compliant suitable constrained branches are shown. Among them, each branch has 3 DOF. As shown in Fig. 8a, the L1 compliant suitable constrained branch only has a flexible straight beam. The flexible beam provides three constrained forces, including two parallel constrained forces. The two parallel constrained forces are equivalent to one constrained force and one constrained moment. Thus, this branch can achieve 2R1T motion. Similarly, as shown in Fig. 8b, the L2 compliant suitable constrained branch has two flexible straight beams, and those two flexible straight beams provide two constrained forces and one constrained moment. Thus, the L2 compliant suitable constrained branch can achieve 2R1T motion. As shown in Fig. 8c, the PU compliant suitable constrained beam has a flexible prismatic hinge and a flexible universal joint. The branch can achieve $2 \mathrm{R} 1 \mathrm{~T}$ motion.

Through steps 1-5, a series of 2R1T compliant suitable constrained branches are synthesized, and some of them are 


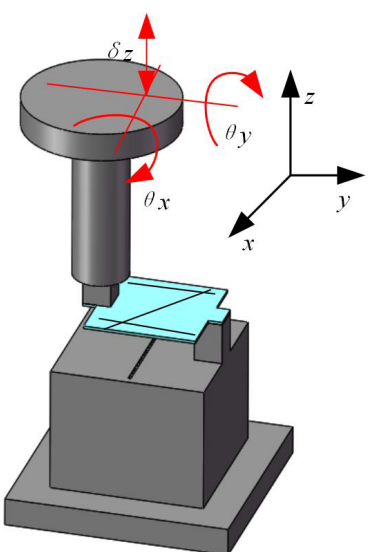

(a)

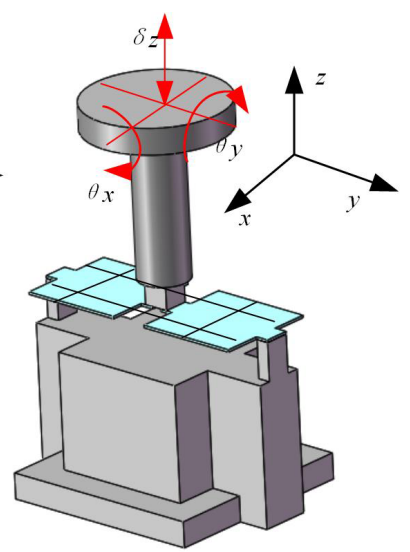

(b)

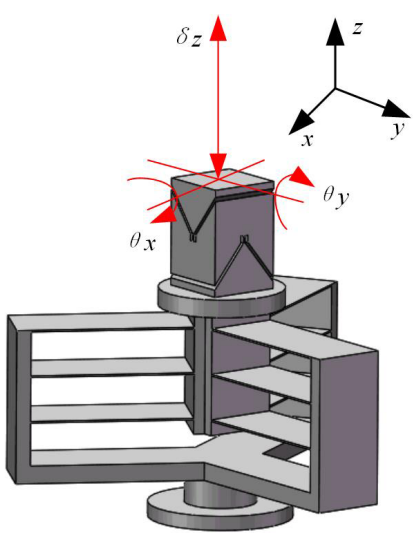

(c)

Figure 8. (a) L1 compliant suitable constrained branch. (b) L2 compliant suitable constrained branch. (c) PU compliant suitable constrained branch.

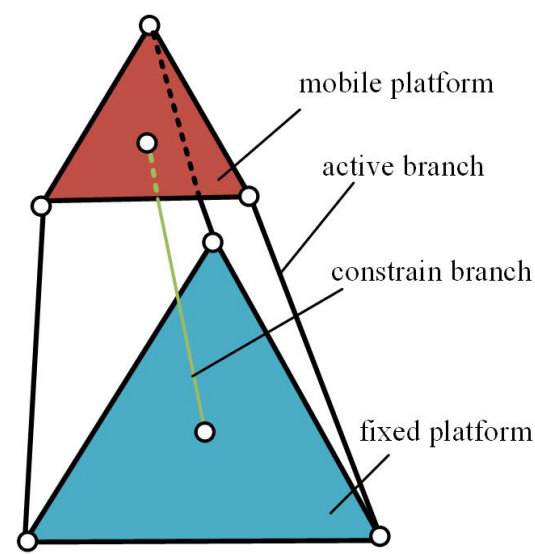

(a)

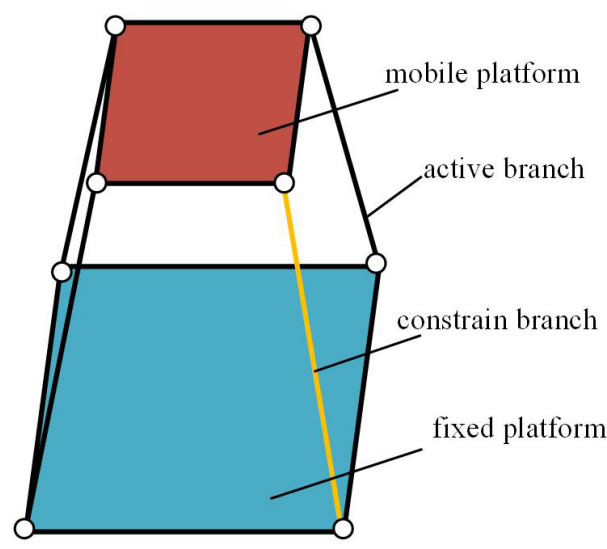

(b)

Figure 9. The two different arrangements for the compliant mechanisms. (a) Axis symmetric. (b) Plane symmetric.

Table 4. Some of the new configurations of compliant parallel mechanism.

\begin{tabular}{llllll}
\hline & & PUS & UPS & SUP & PSS \\
\hline Axis symmetrical arrangement & L & 3 PUS/L(1) & 3 UPS/L(1) & 3 SUP/L(1) & 3 PSS/L(1) \\
& PU & 3 PUS/PU(1) & 3 UPS/PU(1) & 3 SUP/PU(1) & 3 PSS/PU(1) \\
& PRR & 3 PUS/PRR(1) & 3 UPS/PRR(1) & 3 SUP/PRR(1) & 3 PSS/PRR(1) \\
& L2 & 3 PUS-L2(1) & 3 UPS/L2(1) & 3 SUP/L2(1) & 3 PSS/L2(1) \\
\hline Plane symmetrical arrangement & L & 3 PUS/L(2) & 3 UPS/L(2) & 3 SUP/L(2) & 3 PSS/L(2) \\
& PU & 3 PUS/PU(2) & 3 UPS/PU(2) & 3 SUP/PU(2) & 3 PSS/PU(2) \\
& PRR & 3 PUS/PRR(2) & 3 UPS/PRR(2) & 3 SUP/PRR(2) & 3 PSS/PRR(2) \\
& L2 & 3 PUS-L2(2) & 3 UPS/L2(2) & 3 SUP/L2(2) & 3 PSS/L2(2) \\
\hline
\end{tabular}




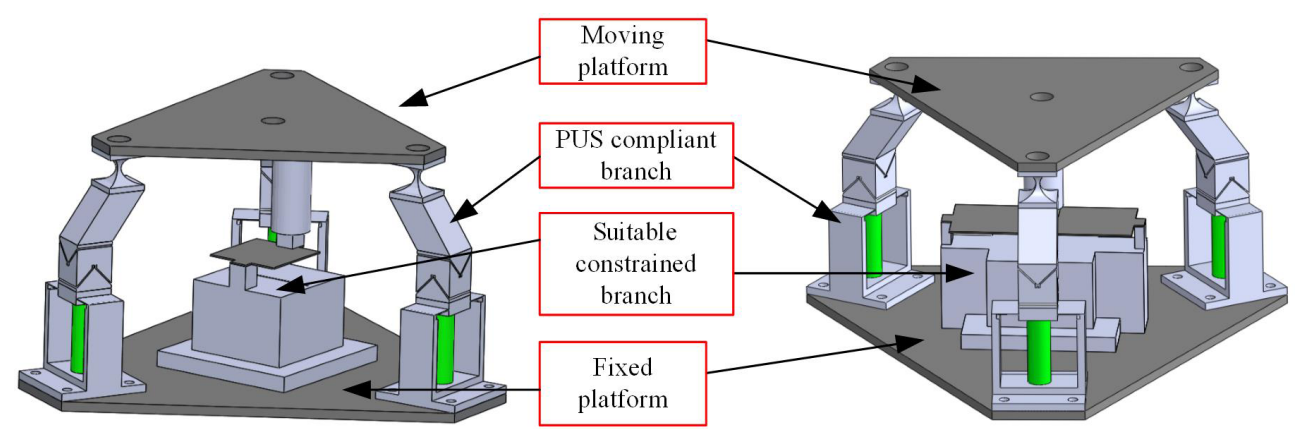

(a)

(b)

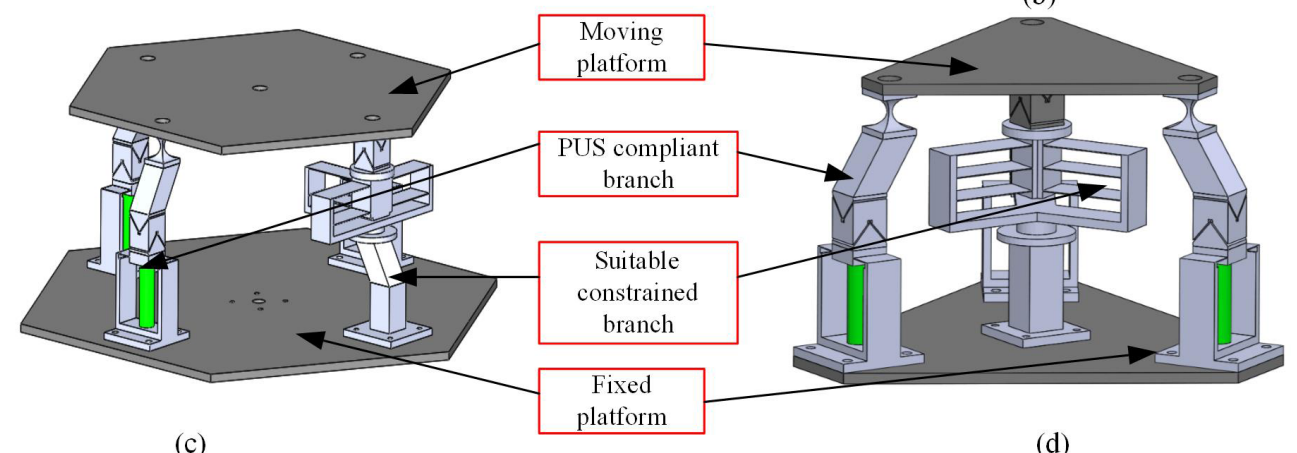

(c)

(d)

Figure 10. The four new CPMs for the suitable constrained branch. (a) 3 PUS/L(1) CPM. (b) 3 PUS/L2(1) CPM. (c) 3 PUS/PU(2) CPM. (d) 3 PUS/PU(1) CPM.

illustrated in Table 3. For L1 as an example, L denotes the flexible straight beam, and 1 denotes the number of the flexible straight beam.

\subsection{Synthesis of the high precision CPM}

Based on the new type synthesis approach, the number of compliant suitable constrained branches and compliant active branches, respectively, need to been determined. After the synthesis of compliant branches is completed, one compliant suitable constrained branch and two compliant active branches are selected by Eqs. (7) and (8). According to the high-precision requirement in Sect. 3.1, the symmetrical arrangement is selected.

Thus, in order to obtain a 2R1T motion CPM with high precision, this section proposes two special symmetrical arrangements. The first symmetrical arrangement can be called the axis symmetrical arrangement. It is considered as two separated components, where three identical compliant active branches are distributed at $120^{\circ}$ around the edge of two platforms, and a compliant suitable constrained branch connects to the center of two platforms. Its structure diagram is shown in Fig. 9a. The second symmetrical arrangement can be called the plane symmetrical arrangement. As shown in Fig. 9b, its structure diagram shows that three identical compliant active branches and one compliant suitable constrained branch are distributed at $90^{\circ}$ around the edge of two platforms.

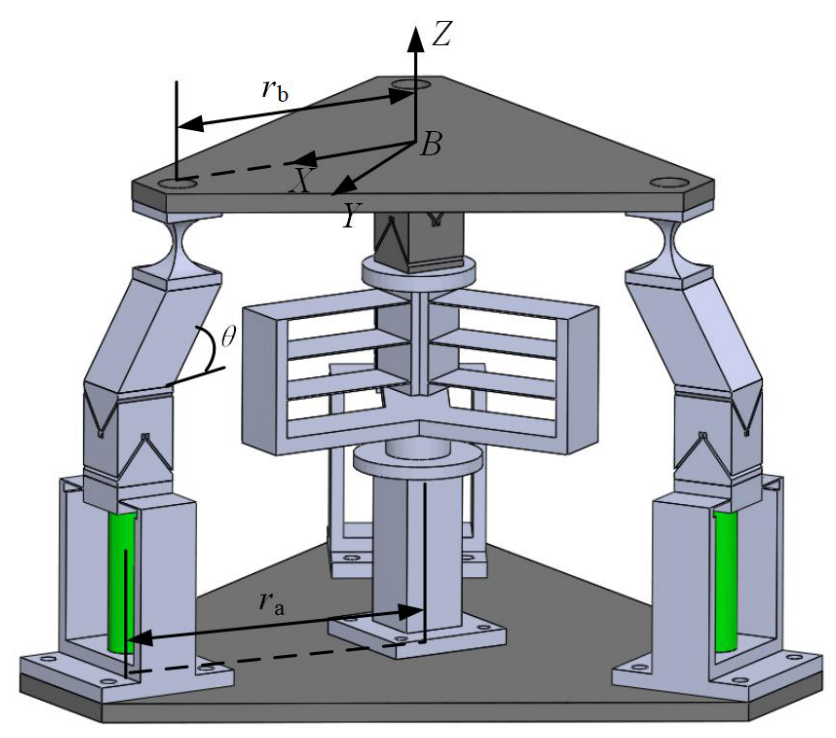

Figure 11. 3 PUS/PU(1) compliant parallel mechanism.

As a result, based on the synthesized compliant active branches and compliant suitable constrained branches, the arrangements are determined. The axis symmetrical (Zhang et al., 2011) and the plane symmetrical arrangements (Pakzad et al., 2019) can achieve high precision when they move in a principal motion direction. As shown in Table 4, a series of the high-precision and high-stiffness CPMs with a suit- 


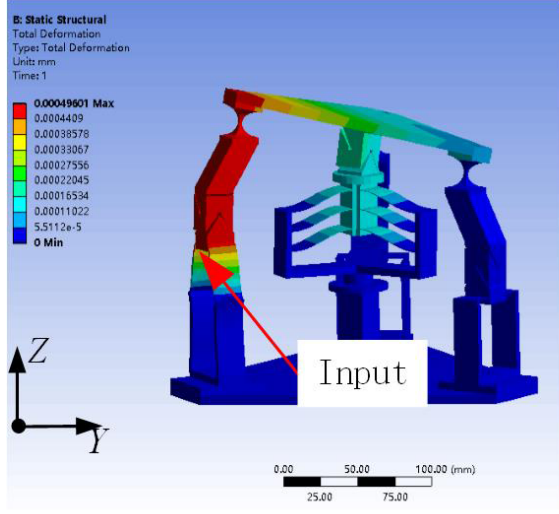

(a)

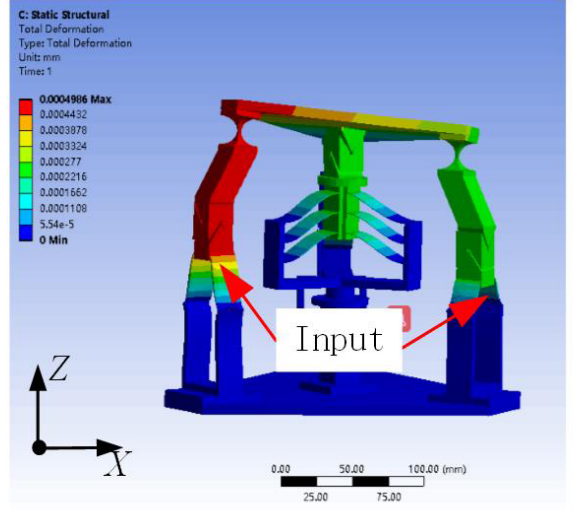

(b)

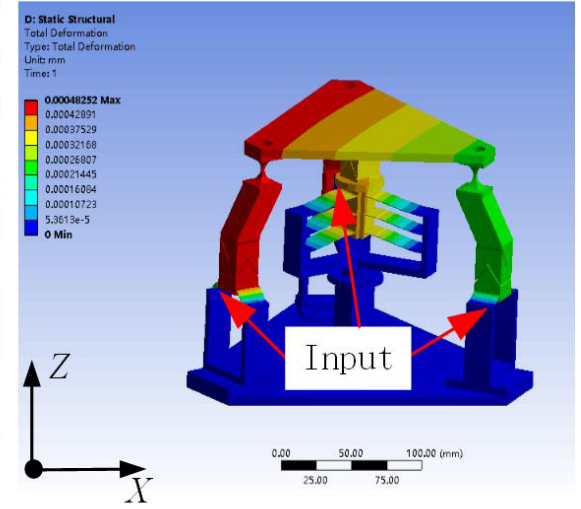

(c)

Figure 12. The deformation distribution, with (a) one actuator, (b) two actuators, and (c) three actuators, respectively.

able constrained branch are proposed through this symmetrical arrangement, and they are illustrated in Fig. 10.

As shown in Table 4, the new 3 PUS/PU(1) compliant parallel mechanism is proposed. For the $3 \mathrm{PUS} / \mathrm{PU}(1)$ compliant parallel mechanism as the example, PUS denotes the PUS compliant active branch, PU denotes the PU compliant suitable constrained branch, and (1) denotes the axis symmetrical arrangement.

In Fig. 10, four kinds of CPMs are demonstrated. Among them, each mechanism has four compliant branches, and the 6 DOF compliant active branches are the PUS compliant branch. As shown in Fig. 10a, 3 PUS/L(1) CPM is an axis symmetrical CPM. The suitable constrained branch has only a narrow flexible straight beam and is applies two constraining forces along the $x$ axis and the $y$ axis and one constraining moment around the $z$ axis on the moving platform. Thus, the new 3 PUS/L1(1) CPM can achieve 2R1T motion. As shown in Fig. 10b, 3 PUS/L2(1) CPM is an axis symmetrical CPM. The suitable constrained branch has two narrow flexible straight beams. The two beams ensure the co-planar plane and apply two constraining forces along the $x$ axis and the $y$ axis and one constraining moment around the $z$ axis on the moving platform. Thus, the new 3 PUS/L2(1) CPM can achieve 2R1T motion. As shown in Fig. 10c, 3 PUS/PU(2) $\mathrm{CPM}$ is an plane symmetrical CPM. The suitable constrained branch has a flexible prismatic hinge and a flexible universal joint. The branch applies two constraining forces along the $x$ axis and the $y$ axis and one constraining moment around the $z$ axis on the moving platform. Similarly, as shown in Fig. 10d, 3 PUS/PU(1) CPM has a suitable constrained branch. The branch applies two constraining forces along the $x$ axis and the $y$ axis and one constraining moment around the $z$ axis on the moving platform.

\subsection{DOF analysis of the CPM}

In order to verify the correctness of the proposed synthesis approach, the DOF of the synthesized mechanism is analyzed. In this section, taking the 3 PUS/PU(1) compliant parallel mechanism as the example, a finite element simulation (Zheng et al., 2015; M. Wang et al., 2019) is carried out to validate the DOF and the motion characteristic of $3 \mathrm{PUS} / \mathrm{PU}(1)$ compliant parallel mechanism, as shown in Fig. 11.

For the finite element model (FEM) of the 3 PUS/PU(1) compliant parallel mechanism, let the circumradius of the moving platform, $r_{b}$, be $150 \mathrm{~mm}$, the circumradius of the fixed platform, $r_{a}$, be $200 \mathrm{~mm}$, and the intersection angle of the compliant active branch with the fixed platform, $\theta$, be $60^{\circ}$. The material is titanium alloy, with a low density and high strength. The material properties are identified, where Young's modulus $E$ is $96 \mathrm{GPa}$, Poisson's ratio $\mu$ is 0.36 , and the density $\rho$ is $4620 \mathrm{~kg} \mathrm{~m}^{-3}$.

For the 3 PUS/PU(1) compliant parallel mechanism, the von Mises resultant deformations will be obtained based upon different inputs of the three actuators. One actuator is turned on, two actuators are turned on, and three actuators are turned on, respectively, and the results are shown in Fig. 12.

The deformation results of the 3 PUS/PU(1) compliant parallel mechanism are illustrated in Fig. 12. It can be clearly seen that the moving platform rotates around the $x$ axis, as illustrated in Fig. 12a, the moving platform rotates around the $y$ axis, as illustrated in Fig. 12b, and the moving platform translations along the $z$ axis, as illustrated in Fig. 12c. Thus, the 3 PUS/PU(1) compliant parallel mechanism can achieve 2R1T motion. The results demonstrate the correctness and feasibility of the proposed synthesized approach for CPM with a suitable constrained branch. 


\section{Conclusion}

In this paper, a systematic methodology for a type synthesis of a 2R1T compliant parallel mechanism with a suitable constrained branch is introduced. The whole type synthesis principle is built upon the PRBM approach and the FACT approach, which is also combined with the principle of symmetry. The criteria for the type synthesis of the CPM with a suitable constrained branch is introduced, and the process of the type synthesis is described in detail. A series of $2 \mathrm{R} 1 \mathrm{~T}$ CPMs with a suitable constraint branch are proposed. Using the finite element simulation, the DOF of the synthesized mechanism is analyzed. The results show that the type synthesis approach is corrected and enables the type synthesis to be simple and effective. These synthesized high-precision and high-stiffness mechanisms have potential applications to space posture adjustments for the space optical load in the aerospace field.

\section{Appendix A: Nomenclature}

$\begin{array}{ll}\text { CM } & \text { Compliant mechanism } \\ \text { CPM } & \text { Compliant parallel mechanism } \\ \text { CS } & \text { Constraint space } \\ \text { DOF } & \text { Degree(s) of freedom } \\ \text { FACT } & \text { Freedom and constraint topology } \\ \text { FBB } & \text { Fundamental building block } \\ \text { FEM } & \text { Finite element model } \\ \text { FS } & \text { Freedom space } \\ \text { MEMS } & \text { Micro electro mechanical system } \\ \text { PM } & \text { Parallel mechanism } \\ \text { PRBM } & \text { Pseudo-rigid-body model } \\ \text { SCB } & \text { Suitable constrained branch } \\ \text { SMs } & \text { Serial mechanisms }\end{array}$

Data availability. All the data used in this paper can be obtained upon request to the corresponding author.

Author contributions. YZ and SL conceived the presented idea. YZ established an overall research framework and the model. SJ and LY assisted with theory and FEM. All the authors read and approved the final paper.

Competing interests. The contact author has declared that neither they nor their co-authors have any competing interests.

Disclaimer. Publisher's note: Copernicus Publications remains neutral with regard to jurisdictional claims in published maps and institutional affiliations.
Acknowledgements. We are grateful for the financial support from the National Natural Science Foundation of China (grant no. 51775475) and the Military and Civilian Integration Project of Hebei Province (grant no. 2020B030).

Financial support. This research has been supported by the National Natural Science Foundation of China (grant no. 51775475) and the Military and Civilian Integration Project of Hebei Province (grant no. 2020B030).

Review statement. This paper was edited by Peng Yan and reviewed by three anonymous referees.

\section{References}

Bhargav, S. D. B., Jorapur, N., and Ananthasuresh, G. K.: Microscale composite compliant mechanisms for evaluating the bulk stiffness of MCF-7 cells, Mech. Mach. Theory, 91, 258-268, https://doi.org/10.1016/j.mechmachtheory.2015.04.002, 2015.

Bilancia, P., Berselli, G., Bruzzone, L., and Fanghella, P.: A CAD/CAE integration framework for analyzing and designing spatial compliant mechanisms via pseudo-rigidbody methods, Robot. Cim.-Int. Manuf., 56, 287-302, https://doi.org/10.1016/j.rcim.2018.07.015, 2018.

Blanding, D. L.: Exact constraint: machine designusing kinematic principles, New York, NY, ASME Press, 1999.

Bowen, C. R., Kim, H. A., Weaver, P. M., and Dunn, S.: Piezoelectric and ferroelectric materials and structures for energy harvesting applications, Energ. Environ. Sci., 7, 25-44, https://doi.org/10.1039/c3ee42454e, 2013.

Cannon, J. R. and Howell, L. L.: A compliant contactaided revolute joint, Mech. Mach. Theory, 40, 1273-1293, https://doi.org/10.1016/j.mechmachtheory.2005.01.011, 2005.

Chen, G. and Ma, F.: Kinetostatic modeling of fully compliant bistable mechanisms using Timoshenko beam constraint model, J. Mech. Design, 137, 1-22, https://doi.org/10.1115/1.4029024, 2015.

Chen, S. L., Ling, M. X., and Zhang, X. N.: Design and experiment of a millimeter-range and high-frequency compliant mechanism with two output ports, Mech. Mach. Theory, 126, 201-209, https://doi.org/10.1016/j.mechmachtheory.2018.04.003, 2018.

Chen, Y. C., Joffre, D., and Avitabile, P.: Underwater dynamic response at limited points expanded to full-field strain response, Int. J. Vib. Acoust., 140, 051016-01-051016-09, https://doi.org/10.1115/1.4039800, 2018.

Chen, Y. C., Mendoza, A. E., and Griffith, D.: Experimental and numerical study of high-order complex curvature mode shape and mode coupling on a three-bladed wind turbine assembly, Mech. Syst. Signal Pr., 160, 107873-1-107873-25, https://doi.org/10.1016/j.ymssp.2021.107873, 2021.

Chen, Z. H., Zhang, Y. B., and Wang, K. M.: Design and analysis of a novel planar translational parallel robotic mechanism with three limbs, IOP Conf. Ser.-Mat. Sci., 646, 012004-1-012004-7, https://doi.org/10.1088/1757-899X/646/1/012004, 2019.

Clark, L., Shirinzadeh, B., Tian, Y., and Yao, B.: Development of a passive compliant mechanism for measurement of mi- 
cro/nanoscale planar 3-DOF motions, IEEE-ASME T. Mech., 21, 1222-1232, https://doi.org/10.1109/TMECH.2015.2503728, 2016.

Cui, X. L., He, Z. Q., Huang, B., Chen, Y. C., Du, Z. X., and Qi, W.: Study on the effects of wheel-rail friction selfexcited vibration and feedback vibration of corrugated irregularity on rail corrugation, Wear, 477, 203857-01-203857-14, https://doi.org/10.1016/j.wear.2021.203854, 2021.

da Silva, G. A., Beck, A. T., Sigmund, O.: Topology optimization of compliant mechanisms considering stress constraints, manufacturing uncertainty and geometric nonlinearity, Comput. Method. Appl. M., 365, 112972 https://doi.org/112972.10.1016/j.cma.2020.112972, 2020.

Emmendoerfer, H., Fancello, E. A., and Silva, E. C. N.: Stress-constrained level set topology optimization for compliant mechanisms, Comput. Method. Appl. M., 362, 112777, https://doi.org/10.1016/j.cma.2019.112777, 2020.

Hopkins, J. B. and Culpepper, M. L.: Synthesis of multi-degree of freedom, parallel flexure system concepts via freedom and constraint topology (FACT) - Part I: Principles, Precis. Eng., 34, 259-270, https://doi.org/10.1016/j.precisioneng.2009.06.008, 2010a.

Hopkins, J. B. and Culpepper, M. L.: Synthesis of multi-degree of freedom, parallel flexure system concepts via freedom and constraint topology (FACT) - Part I: Principles, Precis. Eng., 34, 271-278, https://doi.org/10.1016/j.precisioneng.2009.06.007, 2010 b.

Hopkins, J. B. and Culpepper, M. L.: Synthesis of precision serial flexure systems using freedom and constraint topologies (FACT), Precis. Eng., 35, 638-649, https://doi.org/10.1016/j.precisioneng.2011.04.006, 2011.

$\mathrm{Hu}, \mathrm{X}$. Y. and Liu, H. Z.: Type synthesis and analysis of class of actuated metamorphic mechanisms with suitable constrained branch, Trans. Chin. Soc. Agric. Mach., 51, 383-390, https://doi.org/10.6041/j.issn.1000-1298.2020.01.042, 2020.

Lang, S. H., Xin, H. B., and Yu, Z. G.: Simulation of kinematicaccuracy of a 6-DOF serial robotic mechanism based on screwtheory, in: 15th IEEE International Conference on AdvancedRobotics and Its Social Impacts (IEEE ARSO), 31 October-2November 2019, Beijing, China, https://doi.org/10.1109/ARSO46408.2019.8948710, 2019.

Li, S., Zhou, Y., Shan, Y., Chen, S., and Han, J.: Synthesis method of two translational compliant mechanisms with redundant actuation, Mech. Sci., 12, 983-995, https://doi.org/10.5194/ms-12983-2021, 2021.

Li, Y. Q., Zhang, Y., and Zhang, L. J.: A new method for type synthesis of 2R1T and 2T1R 3-DOF redundant actuated parallel mechanisms with closed loop units, Chin. J. Mech. Eng.-En., 33, 2-24, https://doi.org/10.1186/s10033-020-00487-7, 2020.

Liang, Y., Sun, K., and Cheng, G. D.: Discrete variable topology optimization for compliant mechanism design via sequential approximate integer programming with trust region (SAIP-TR), Struct. Multidiscip. O., 62, 2851, https://doi.org/10.1007/s00158-021-03083-y, 2020.

Lobontiu, N.: Compliant Mechanisms: Design of Flexure Hinges, CRC Press, Boca Raton, FL, USA, https://doi.org/10.1201/9781420040272, 2002.

Jia, M., Jia, R. P., and Yu, J. J.: A compliance-based parameterization approach for type synthesis of flexure mechanisms, J. Mech. Robot., 7, 031014-1-031014-12, https://doi.org/10.1115/1.4028932, 2015.

Kozuka, H., Arata, J., Okuda, K., Onaga, A., Ohno, M., Sano, A., and Fujimoto, H.: A bio-inspired compliant parallel mechanism for high-precision robots, IEEE International Conferenceon Robotics and Automation (ICRA), 14-18 May 2012, Saint Paul, Minnesota, USA, https://doi.org/10.1109/ICRA.2012.6224873, 2012.

Moon, Y., Crane, C. D., and Roberts, R. G.: Reverse kinetostatic analysis and stiffness synthesis of a spatial tensegritybased compliant mechanism, Mech. Mach. Theory, 70, 320-337, https://doi.org/10.1016/j.mechmachtheory.2013.05.001, 2013.

Pakzad, S., Akhbari, S., and Mahboubkhah, M.: Kinematic and dynamic analyses of a novel 4-DOF parallel mechanism, J. Braz. Soc. Mech. Sci., 41, 2-13, https://doi.org/10.1007/s40430-0192058-3, 2019.

Pinskier, J., Shirinzadeh, B., Clark, L., and Qin, Y. D.: Development of a 4-DOF haptic micromanipulator utilizing a hybrid parallel-serial flexure mechanism, Mechatronics, 50, 55-68, https://doi.org/10.1016/j.mechatronics.2018.01.007, 2018.

Pucheta, M. A. and Cardona, A.: Design of bistable compliant mechanisms using precision-position and rigid-body replacement methods, Mech. Mach. Theory, 45, 304-326, https://doi.org/10.1016/j.mechmachtheory.2009.09.009, 2010.

Qi, H. M., Zhang, B. J., Zhang, N., Zheng, M. Y., and Chen, Y. C.: Enhanced lateral and roll stability study for a two-axle bus via hydraulically interconnected suspension tuning, Sae. Int. J. Veh. Dyn. St., 3, 5-18, https://doi.org/10.4271/10-03-01-0001, 2018.

Qiu, L. F., Liu, N. N., Chen, M. K., and Yue, X.: Design and performance analysis of nested-LET flexure hinge, Trans. Chin. Soc. Agric. Mach., 49, 420-426, https://doi.org/10.6041/j.issn.1000.1298.2018.05.051, 2018.

Shao, Z. X., Wu, S. L., and Fu, H. Y.: Stiffness analysis of a novel flexible positioning mechanism for largeaperture grating tiling, J. Mech. Eng., 54, 117-125, https://doi.org/10.3901/JME.2018.13.117, 2018.

Wang, G. X., Liu, H. Z., and Yuan, D. N.: Force analysis of spatial 4-SPS/CU parallel mechanism, Trans. Chin. Soc. Agric. Eng., 28, 30-38, https://doi.org/10.3969/j.issn.10026819.2012.22.005, 2012.

Wang, L. T., Li, J., and Zhao, L.: Kinematic analysis and speed control of 3SPS-1S parallel mechanism for end actuator of segmenterector, 12th International Conference on Intelligent Roboticsand Applications, ICIRA 2019, 8-11 August 2019, Shenyang, China, https://doi.org/10.1007/978-3-03027529-7_17, 2019.

Wang, M., Zhang, B. J., Chen, Y. C., Zhang, N., and Zhang, J.: Frequency-based modeling of a vehicle fitted with rollplane hydraulically interconnected suspension for ride comfort and experimental validation, IEEE Access, 8, 1091-1104, https://doi.org/10.1109/ACCESS.2019.2935260, 2019.

Wang, R. Z. and Zhang, X. M.: Optimal design of a planar parallel 3-DOF nanopositioner with multi-objective, Mech. Mach. Theory., 112, 61-83, https://doi.org/10.1016/j.mechmachtheory.2017.02.005, 2017.

Yang, M., Du, Z. J., Chen, F. X., Dong, W., and Zhang, D.: Kinetostatic modelling of a 3-PRR planar compliant parallel manipulator with flexure pivots, Precis. Eng., 48, 323-330, https://doi.org/10.1016/j.precisioneng.2017.01.002, 2017. 
Yang, M., Zhang, C., Yu, H. T., Huang, X. L., Yang, G. L., and Fang, Z. J.: Kinetostatic modeling of redundantly actuated planar compliant parallel mechanism, 12th International Conference on Intelligent Robotics and Applications (ICIRA), 8-11 August 2019, Shenyang, China, https://doi.org/10.1007/978-3030-27526-6_31, 2019.

Yang, Y., Wu, G., and Wei, Y.: Design, modeling, and control of a monolithic compliant $x-y-\theta$ microstage using a double-rocker mechanism, Precis. Eng., 71, 209-231, https://doi.org/10.1016/j.precisioneng.2021.03.014, 2021.

Yao, J. T., Zhao, Y. S., Chen, L. P., Xu, Y. D., and Gu, W. D.: Dynamic analysis and driving force optimization of a 5-DOF parallel manipulator with redundant actuation, Robot. CIM-Int. Manuf., 48, 51-58, https://doi.org/10.1016/j.rcim.2017.02.006, 2017.

Yu, J. J., Li, S. Z., Pei, X., Su, H. J., Hopkins, J. B., and Culpepper, M. L.: Type Synthesis Principle and Practice of Flexure Systemsin the Framework of Screw Theory: Part I General Methodology, Asme International Design Engineering Technical Conferences, 15-18 August 2010, Montreal, Canada, https://doi.org/10.1115/DETC2010-28783, 2010.

Yu, J. J., Li, S. Z., Su, H. J., and Culpepper, M. L.: Screw theory based methodology for the deterministic type synthesis of flexure mechanisms, J. Mech. Robot., 3, 1194-1204, https://doi.org/10.1115/1.4004123, 2011.

Yu, J. J., Lu, D. F., and Xie, Y.: Constraint design principle oflargedisplacement flexure systems, in: 2014 International Conference on Manipulation, Manufacturing and Measurement onthe Nanoscale (3M-NANO), 27-31 October 2014, Taipei, Taiwan, https://doi.org/10.1109/3M-NANO.2014.7057361, 2014.

Yu, J. J., Hao, G. B., Chen, G. M., and Bi, S. S.: State of-art of compliant mechanisms and their applications, J. Mech. Eng., 51, 53-68, https://doi.org/10.3901/JME.2015.13.053, 2015.
Yu, J. J., Lu, D. F., and Hao, G. B.: Design and analysis of a compliant parallel pan-tilt platform, Meccanica, 51, 1559-1570, https://doi.org/10.1007/s11012-015-0116-1, 2016.

Yun, Y. and Li, Y. M.: Optimal design of a 3-PUPU parallel robot with compliant hinges for micromanipulation in a cubic workspace, Robot. CIM-Int. Manuf., 27, 977-985, https://doi.org/10.1016/j.rcim.2011.05.001, 2011.

Zhang, D., Gao, Z., and Fassi, I.: Design optimization of a spatial hybrid mechanism for micromanipulation, Int. J. Mech. Mater. Des., 7, 55-70, https://doi.org/10.1007/s10999-011-91493, 2011.

Zhang, X. M., Hu, K., Wang, N. F., and Zhang, B.: Multi-objective topology optimization of multiple material compliant mechanisms based on parallel strategy, J. Mech. Eng., 52, 1-8, https://doi.org/10.3901/JME.2016.19.001, 2016.

Zhang, H. Q. and Fang, H. R.: Performance analysis of a redundantly actuated parallel manipulator with suitable constraint branch, J. Beijing Jiaotong Univ., 42, 133-138, https://doi.org/10.11860/j.issn.1673-0291.2018.01.020, 2018.

Zhao, H. Z., Bi, S. S., and Yu, J. J.: A novel compliant linear-motion mechanism based on parasitic motion compensation, Mech. Mach. Theory, 50, 15-28, https://doi.org/10.1016/j.mechmachtheory.2011.11.009, 2012.

Zheng, M. Y., Peng P., Zhang, B. J., Zhang, N., Wang, L. F., and Chen, Y. C.: A new physical parameter identification method for two-axis on-road vehicles: simulation and experiment, Shock. Vib., 2015, 1-9, https://doi.org/10.1155/2015/191050, 2015. 